\title{
Potential Benefit of Metformin as Treatment for Colon Cancer: the Evidence so Far
}

\author{
Azza Ali Abdelsatir ${ }^{1}$, Nazik Elmalaika Husain ${ }^{2}$, Abdallah Tarig Hassan ${ }^{3}$, Wadie \\ M Elmadhoun ${ }^{4}$, Ahmed O Almobarak ${ }^{5}$, Mohamed H Ahmed ${ }^{6 *}$
}

\begin{abstract}
Metformin is known as a hypoglycaemic agent that regulates glucose homeostasis by inhibiting liver glucose production and increasing muscle glucose uptake. Colorectal cancer (CRC) is one of the most common cancers worldwide, with about a million new cases diagnosed each year. The risk factors for CRC include advanced age, smoking, black race, obesity, low fibre diet, insulin resistance, and the metabolic syndrome. We have searched Medline for the metabolic syndrome and its relation to CRC, and metformin as a potential treatment of colorectal cancer. Administration of metformin alone or in combination with chemotherapy has been shown to suppress CRC. The mechanism that explains how insulin resistance is associated with CRC is complex and not fully understood. In this review we have summarised studies which showed an association with the metabolic syndrome as well as studies which tackled metformin as a potential treatment of CRC. In addition, we have also provided a summary of how metformin at the cellular level can induce changes that suppress the activity of cancer cells.
\end{abstract}

Keywords: Metformin - colon cancer - obesity and insulin resistance

Asian Pac J Cancer Prev, 16 (18), 8053-8058

\section{Introduction}

Colorectal cancer (CRC) is one of the most common cancers worldwide, with about a million cases diagnosed each year. Multiple risk factors were identified including increasing age, smoking, black race, obesity, low fibre diet, insulin resistance, and the metabolic syndrome. There is sharp increase in incidence of metabolic syndrome worldwide which is defined as the presence of at least three of the following components: increased waist circumference, hypertriglyceridaemia, low high-density lipoprotein (HDL), hypertension, and hyperglycaemia (Kaneko et al.,2014).

Increased body weight and type 2 diabetes have been linked to many types of cancer. This association has primarily been attributed to insulin resistance and cluster factors of metabolic syndrome thought to also play additive carcinogenic roles. In addition, the so-calledWestern diet, rich in processed foods and meats, sedentary lifestyle, and obesity are risk factors for colorectal cancer which may be mediated by hyperinsulinemia (Inoue and Tsugane, 2012). Not only a risk factor for colorectal cancer, excess body weight also increases the risk of esophageal adenocarcinoma, pancreatic cancer, postmenopausal breast cancer, endometrial cancer, kidney cancer, and risk of colorectal cancer. Furthermore, it is probable that obesity increases the risk of gallbladder cancer, and possibly increases the risk of liver cancer as well. Importantly, type 2 diabetes mellitus (DM) has a positive link to specific cancers in different populations that are unrelated to excess body weight. Recent reviews and meta-analyses showed a strong positive association for DM and pancreatic cancer, hepatocellular carcinoma and endometrial cancer. DM also probably increases the risk for biliary tract, bladder, colorectal, oesophageal and breast cancers (Inoue and Tsugane, 2012).

\section{Glucose, insulin resistance and cancer}

Glucose is one source of energy for tumor cells to support growth and proliferation. Tumour cells depend on the intake of amino acids such as glutamine. Glucose uptake is regulated by growth factor signalling in normal non-proliferating cells. Activation of growth factor receptors stimulates changes in intracellular signalling, which in turn modifies metabolic pathways in support of proliferative growth. Hyperglycemia is often wrongly implicated as the sole source of cancer nutrition in patients with diabetes, when in fact cancer cells can thrive using other energy sources promoted by genetic mutations and aberrant intracellular signalling (Handelsman et al., 2013). Insulin has been shown to affect the growth of both normal

${ }^{1}$ Histocenter, Khartoum, Sudan, ${ }^{2}$ Department of Pathology, Faculty of Medicine and Health Sciences, Omdurman Islamic University, ${ }^{4}$ Nile Valley University, Atbara, ${ }^{3}$ Department of Pharmacy, Blue Nile Pharmaceuticals, ${ }^{5}$ Department of Pathology, Faculty of Medicine, University of Medical Sciences and Technology, Khartoum, Sudan, ${ }^{6}$ Department of Medicine, Milton Keynes University Hospital NHS Foundation Trust, Milton Keynes, Buckinghamshire, UK *For correspondence: elziber@yahoo.com 
and neoplastic epithelial cells and to have the capability of promoting mitosis in vitro, directly or indirectly via Insulin-like growth factor 1 (IGF-1). It has been proposed that insulin and insulin-like growth factors may be involved in colon carcinogenesis (Trevisan et al., 2001, Aleksandrova et al., 2011). Insulin acts as a growth factor, and insulin receptors are present in normal and malignant colorectal cells. This action would be potentiated by hyperglycaemia which induces the secretion of insulin (Trevisan et al., 2001).

Hyperinsulinemia may also affect the development of colorectal cancer, indirectly by down-regulating insulinlike growth factor binding proteins (IGFBP-1,2,3), thus enhancing IGF-1 bioavailability (Sandhu et al., 2002). This also happens via insulin binding to IGF-1 receptors (IGF1Rs) (Mulholland et al., 2009). Since IGF-1 is an important mitogen for progression through the cell cycle in addition to its autocrine, paracrine and endocrine actions on cell proliferation and apoptosis, leading to increased cell proliferation, inhibition of apoptosis and promotion of tumour growth(Grimberg and Cohen, 2000). IGF-1 also increases the production of vascular endothelial growth factor (VEGF), an angiogenic factor that may support cancer growth (Warren et al., 1996). The association of IGF-1 and IGFBP-3 levels with increased risk of colorectal adenoma/cancer initially identified through studies of acromegalics, who have chronically increased growth hormone (GH) levels. Excess GH leads to hepatic and peripheral insulin resistance which in turn leads to excess insulin production, causing IGF-1 hypersecretion and low IGFBP-3 levels (Jenkins, 2004).

Giovannucci et al studied the relationships between IGF-1 and IGFBP-3 levels and colorectal cancer in a study of 32,826 women (Giovannucci et al., 2000). They showed that those with higher levels of IGF-1 were at increased risk of intermediate/late-stage colorectal neoplastic adenoma and cancer. The women with higher levels of
IGFBP-3 were at lower risk of intermediate/late-stage of colorectal adenoma and cancer, indicating opposing effects of IGF-1 and IGFBP-3 to colorectal cancer.

William et al analysed the independent role of IGFBP-3 in colorectal cancer (Williams et al., 2000). IGFBP-3 has been shown to enhance p53-dependent apoptosis after DNA damage. Hence, loss of IGFBP-3 could contribute to the development of colorectal adenomas that retain wild-type p53 function through the suppression of p53-dependent apoptotic signals, promoting survival of abnormal cells and tumour-genesis. These studies suggest that IGFBP-3 may have an important role in the regulation of differentiation and apoptosis in human colonic epithelium.

\section{The metabolic syndrome and colorectal cancer}

The metabolic syndrome and visceral obesity have an increasing prevalence worldwide and the prevalence in Europe is estimated to be between $24.6 \%$ and $30.9 \%$ (Pais et al., 2009). Several studies have found an association between metabolic syndrome and CRC (Table 1).

The clinical features of the metabolic syndrome, including obesity, dyslipidaemia, and impaired glucose tolerance, have been linked to an increased risk for colorectal cancer in several recent epidemiological studies (Pais et al., 2009). In these studies, carcinogenesis is linked to hyperinsulinaemia, increased c-peptide, increased body mass index (BMI), high levels of IGF-1, low levels of insulin growth factor binding protein-3, high leptin levels and low adiponectin levels (Pais et al., 2009).

\section{Colorectal cancer and obesity}

Obesity is a growing global health problem, and affects not only adults but also children. Despite the

Table 1. Summary of Studies Demonstrating the association between Diabetes, Insulin Impaired Glucose Tolerance and Colorectal Cancer

\begin{tabular}{|c|c|}
\hline Reference & Main outcome \\
\hline Trevisan et al, 2001 & $\begin{array}{c}\text { Relative risks of colorectal cancer ranging from 1.2 to 2.8 in prospective cohort studies. Studies } \\
\text { have positively correlated colorectal cancer with C-peptide levels, (an insulin-release marker), } \\
\text { 2-hour postchallenge insulin and glucose concentrations, and glycosylated haemoglobin levels. }\end{array}$ \\
\hline Aleksandrova et al, 2011 & $\begin{array}{c}\text { Study suggested that the increased risk of colorectal cancer in diabetics was via the increased } \\
\text { HbA1c level. The lowest incidence of CRC risks occurred in those with HbA1c of lower than 5\% }\end{array}$ \\
\hline Saydah et al, 2003 & $\begin{array}{c}\text { Significant risk of colorectal cancer mortality with impaired glucose tolerance, but not diabetes } \\
\text { (RR 1.87 vs 1.13) }\end{array}$ \\
\hline Khaw et al, 2003 & $\begin{array}{c}\text { HbA1c significantly predicted incident colorectal cancer independent of diabetes. An increase in } \\
\text { HbAlc of 1\% was associated with 33\% increase in colorectal cancer risk. }\end{array}$ \\
\hline Shin HY, 2014 & The incidence of CRC positively correlated with fasting plasma glucose \\
\hline Vu H, 2014 & $\begin{array}{c}\text { DM was associated with higher risk of colorectal adenomas in patients ages 40-49 years. These } \\
\text { subjects harbored as many adenomas as those at the standard screening age of 50-59 years with- } \\
\text { out DM. }\end{array}$ \\
\hline Rampal s et al, 2014 & $\begin{array}{c}\text { Increasing levels of glucose, HOMA values, levels of hemoglobin A1c and C-peptide, and meta- } \\
\text { bolic syndrome are significantly associated with the prevalence of adenomas }\end{array}$ \\
\hline Huang HE et al, 2014 & $\begin{array}{c}\text { Diabetes, but not pre-diabetes, was associated with a higher risk of advanced adenomatous } \\
\text { polyps. In addition, both diabetes and pre-diabetes were important correlates of non-advanced } \\
\text { adenomatous polyps. }\end{array}$ \\
\hline
\end{tabular}


fact that obesity is linked to diabetes and cardiovascular disease there are no indicators that the epidemic of obesity is decreasing. Although awareness has become more widespread, obesity prevalence in western countries is high. In the USA alone, $71 \%$ of men and $62 \%$ of women are overweight (Ogden et al., 2006). Similar trends were also observed in UK as $65 \%$ of men and $56 \%$ of women are overweight (Zaninotto et al., 2010). The association between obesity and cancer is well documented. For instance, obesity is implicated to cause $14 \%$ of all tumours, including gynaecological malignancies, colon cancer, kidney cancer and less commonly leukaemia, multiple myeloma, and non-Hodgkin's lymphoma (Donohoe et al., 2014).

The mechanisms of carcinogenesis due to obesity are not well understood. Chronic hyperinsulinemia and insulin resistance are thought to be the major factors. Other factors like, insulin-like growth factors, sex steroids, adipokines, obesity-related inflammatory markers, the nuclear factor kappa beta (NF-kappa $\beta$ ) system and oxidative stresses, may also play a role in the development of an environment favourable to cancer growth (Renehan et al., 2008). Type 2 diabetes, which is strongly linked with obesity, leads to increased levels of circulating insulin and non-protein banded IGF-1 that stimulate cellular growth and inhibit apoptosis. Adipokines also play a major role in this process. Excess leptin and (PAI-1) in addition to decreased adiponectin stimulate cellular growth and angiogenesis. PAI- 1 also promotes cell migration by remodelling the extracellular matrix (Hillon et al., 2010).

Several studies showed a direct association between obesity and colorectal cancer. For instance, a metaanalysis of thirty prospective studies done by Larsson SC, Wolk A, Larsson S, Wolk A (2007), stated that a 5-unit increase in BMI was directly proportional to an increased risk of colon cancer in both men (RR: 1.30;
95\% CI: $1.25,1.35$ ) and women (RR: $1.12 ; 95 \%$ CI: 1.07 , $1.18)$, but the association was stronger in men $(\mathrm{P}<0.001)$ and not consistent in women. Obesity is associated with rectal cancer more than colonic cancer in men (RR: 1.12; 95\% CI: $1.09,1.16$ ) but not in women (RR: 1.03 ; 95\% CI: $0.99,1.08)$. Colon cancer risk is associated with increased waist circumference (per 10-cm increase) in both men and women (RR: 1.33; 95\% CI: 1.19, 1.49) (RR: 1.16; $95 \%$ CI: $1.09,1.23)$ respectively, and is also associated with increased waist-hip ratio (per 0.1-unit increase) in both men and women (RR: 1.43 ; 95\% CI: $1.19,1.71$ ) (RR: $1.20 ; 95 \%$ CI: $1.08,1.33$ ) respectively. In sum, the study revealed the relation between obesity and colon and rectal cancer risk is gender and site specific (Larsson and Wolk, 2007).

Interestingly, Na SY, Myung SJ in 2012 hypothesized that obesity is a strong risk factor for CRC and that obese and over-weight individuals are 1.5 times more likely than normal-weight individuals to develop colonic cancer. Furthermore, obese males are more liable to develop CRC than their female counterparts. Na SY, Myung SJ also found that exercise and dietary control do not only reduce risk of CRC but also improve the prognosis and outcome of the disease (Na and Myung, 2012). On the whole, there is general agreement in the literature about the link between obesity and the incidence of colorectal cancer with diverse but not yet fully understood pathways (Kim, 2012). A cross sectional study done in Korea involved 18,085 healthy Korean adults (39.1 \pm 6.7 years) defined as metabolically healthy over-weight (MHOW) individuals after medical evaluation. The aim of the study was to compare the prevalence of high risk adenoma (CRA) ( defined as any adenoma over $1 \mathrm{~cm}, 3$ or more adenomas, adenoma with a villous component, or high-grade dysplasia) among the study population and metabolically healthy with normal weight. Surprisingly, after adjusting

Table 2. Some Studies Demonstrating the Association between the Metabolic Syndrome and Colorectal Cancer

\begin{tabular}{|c|c|}
\hline Reference & Main outcome \\
\hline Aleksandrova et al, 2011 & $\begin{array}{c}\text { Among individual components of the metabolic syndrome, abdominal obesity was associated } \\
\text { with colon cancer (RR }=1.51 ; 95 \% \text { CI: 1.16-1.96), whereas abnormal glucose metabolism was } \\
\text { associated with both colon (RR }=2.05 ; \text { 95\% CI: 1.57-2.68) and rectal cancer (RR }=2.07 ; 95 \% \\
\text { CI: 1.45-2.96 }\end{array}$ \\
\hline Calle et al, 2003 & The relative risk of colorectal cancer showed a dose-response to an increasing BMI. \\
\hline Ahmed et, 2006 & $\begin{array}{c}\text { The metabolic syndrome (>3 components vs 0 components) had a positive association with } \\
\text { colorectal cancer incidence }\end{array}$ \\
\hline Trevisan et al, 2001 & $\begin{array}{c}\text { This study demonstrated that the relative risk of death from colorectal cancer was increased in } \\
\text { cluster analysis compared to glucose level alone. }\end{array}$ \\
\hline Colangelo et al, 2002 & $\begin{array}{c}\text { There was 35\% increased risk of colorectal cancer associated with high blood pressure. It also } \\
\text { found that clustering of features of the metabolic syndrome significantly increased the risk of } \\
\text { associated colorectal cancer. }\end{array}$ \\
\hline Kabat et al, 2012 & $\begin{array}{c}\text { Showed that an increase in glucose level and systolic blood pressure was associated with high } \\
\text { risk of colon cancer }\end{array}$ \\
\hline Chiu HM et al, 2015 & $\begin{array}{c}\text { Metabolic syndrome is risk factor for advanced colon ca and should be included in risk stratifica- } \\
\text { tion }\end{array}$ \\
\hline Lin XF, 2014 & $\begin{array}{c}\text { Fatty liver is hepatic component of metabolic syndrome is regarded as risk factor for colon } \\
\text { cancer }\end{array}$ \\
\hline Esposito et al, 2013 & $\begin{array}{c}\text { Systematic review showed that the risk of colon cancer conveyed by the full syndrome is not } \\
\text { superior to the sum of its parts. }\end{array}$ \\
\hline
\end{tabular}


for many variables such as age, sex, smoking, alcohol drinking, exercise, family history of colorectal cancer, education, and use of analgesic and aspirin, the study found that MHOW individuals were both more low-risk and more high-risk for CRA, $(\mathrm{OR}=1.44 ; 95 \%$ confidence interval (CI), 1.23-1.69 and $\mathrm{OR}=1.62 ; 95 \% \mathrm{CI}, 1.09-2.41$, respectively), compared to the healthy normal weight population. This finding indicates that obesity by itself is a risk factor for pre-cancer conditions in the absence of hyperglycaemic state (Yun et al., 2013). Exercise may in part prevent the incidence of CRC. Sanchez NF et al showed that patients who exercised regularly had less polyps $(25.3 \%$ vs. $33.2 \%, \mathrm{P}=0.008)$ and adenomas $(13.8$ vs. $18.9 \%, \mathrm{P}=0.03$ ) compared to those who did not exercise (Sanchez et al., 2012).

\section{Diabetes, insulin resistance and colorectal cancer}

Several studies have shown that abnormal glucose metabolism is associated with an increased risk of colorectal cancer (Khaw et al., 2004; Hsu et al., 2012). Table 2 provides a summary of studies addressing the association between diabetes, insulin resistance and colorectal cancer.

\section{Metformin as a potential treatment of colon cancer}

Metformin is known as a hypoglycaemic agent that regulates glucose homeostasis by inhibiting liver glucose production and increasing muscle glucose uptake. Many studies have been carried out investigating the effect of metformin on colorectal cancer (CRC) mortality and morbidity. In a larger prospective, metformin is said to be a magical drug with many beneficial effects on many aspects (Saydah et al., 2003; Khaw et al., 2004; Kalaany and Sabatini, 2009; Mahmood, 2013; Zhang et al., 2013; Vuet al., 2014; Rampal et al., 2014; Huang et al., 2014; Wang and Zhang, 2014; Li et al., 2015).

In a cohort study carried out by UK prospective diabetes study concerning the advantages of metformin on type II diabetic patients, metformin was found to play a positive role in lipid disorders, antipsychotic induced weight gain, HIV lipodystrophy syndrome, to improve markers of metabolic syndrome improves fertility of females with polycystic ovary syndrome and delays aging, aging-related disorders and inflammatory-related disorders (Mahmood et al., 2013).

The mechanism of metformin as an antineoplastic agent is not well understood. A preclinical study showed that metformin inhibits insulin-independent growth and xenograft tumour growth of cells carrying the gain-offunction H1047R mutation of the PI3KCAgene (Kalaany and Sabatini, 2009). It is now known that metformin activates adenosine monophosphate kinase (AMPK) and inhibits the mammalian target of rapamycin (mTOR)/ S6K1 pathway, leading to suppressed colonic epithelial proliferation and reduced colonic polyp formation, this function applies to both chemically-induced carcinogenesis and APC-mutated carcinogenesis. In spite of these findings, mTOR inhibitor drugs are not yet authorised for colorectal cancer treatment (Wang and Zhang, 2014).

Zhang Yet al studied the synergistic effect of metformin and 5-Flurouracil (5-FU) on the proliferation, cell cycle and apoptosis of CD133+ cancer stem cells of SW620 human colorectal cancer cell lines. In a group of 86 patients with CRC and type II diabetes divided into metformin and non-metformin users, they found a decrease in distant metastasis by $5.6 \%$ vs $21.6 \%(\mathrm{p}=0.035)$. In addition, lower incidence of poorly differentiated adenocarcinoma was noted. Synergistic effect of Metformin and flurouracil (5-FU) reduced CD133+ population as well as B-catenin expression, suggesting that metformin might have a synergistic antineoplastic effect on colorectal Colorectal cancer stem cells, through B-catenin pathway (Zhang et al., 2013b). Interestingly, the simultaneous administration of metformin and vitamin D exhibits synergistic effects against the development of early colon neoplasia ( $\mathrm{Li}$ et al., 2015).

A meta-analysis of 37 studies with 1,535,635 total participants published in 2013, explored the relation of metformin to reduction in cancer incidence and mortality. In this study two groups of diabetic type II patients of metformin users versus non metformin users were compared. The analysis revealed a specific reduction rate for overall cancer incidence among metformin users of 0.73 (95\% CI 0.64-0.83), mortality was 0.62 (95\%CI 0.76$0-89)$. The risk reduction of liver, pancreatic, colorectal and breast cancers in this study was $78 \%, 46 \%, 23 \%$, $6 \%$, respectively. No statistically significant association between metformin and prostate cancer incidence was found. (Zhang et al., 2013a). Interestingly, in metaanalysis models metformin was associated with decreased risk of all-cause cancer mortality among patients with concurrent diabetes. The result was particularly evident in breast cancer RR 0.7 ( $\mathrm{p}=0.003)$, colorectal cancer RR0.7 ( $<<0.001)$, ovarian cancer RR0.44 (p<0.001) and endometrial cancer RR0.49 $(\mathrm{p}=0.001)$ (Zhang and Li, 2014).

The body of evidence to suggest that metformin effectively lowers the risk of CRC among type II diabetic patients is substantial. For instance, another meta-analysis showed a $37 \%$ low risk of colorectal cancer among diabetic type II on metformin compared to non-metformin users RR 0.63 (95\%CI 0.5-0.79 p<0.001). (Zhang et al., 2011). Yet, another meta-analysis showed that metformin administration was associated with an increase in overall survival benefit and the effect of metformin on colorectal cancer is dose related. High dose of metformin lowers colorectal cancer specific mortality (Mei et al., 2014). This dose related benefit of metformin was also seen with colorectal adenoma (Kanadiya et al., 2013).

Cho et al. (2014) showed that metformin reduced the incidence of adenomas that may transform into CRC, and metformin may be useful for the prevention of CRC in patients with type II DM (Cho et al., 2014). It is worth mentioning that in advanced stage of CRC or metastasis metformin showed limited benefit (Lee et al., 2012; Spillane et al., 2014). 


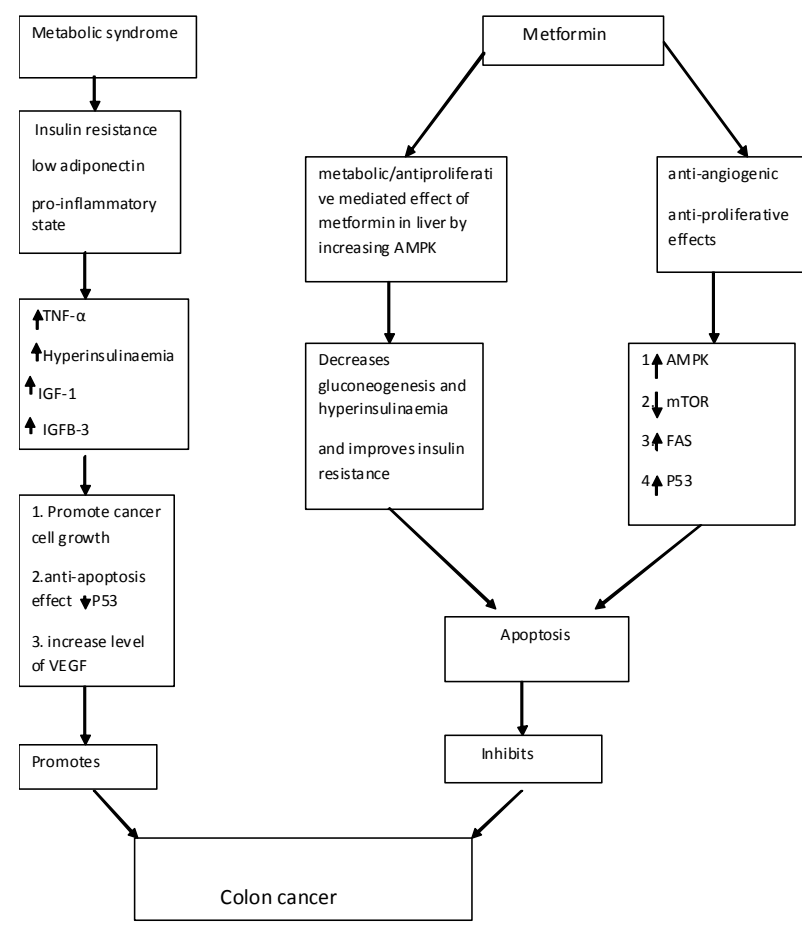

Figure 1. Schemtic Figure of Proposed Antineoplastic Mechanism of Metformin and Role Against Colon Cancer

In contrast to the body of research supporting the decreased risk of CRC among metformin users, other studies found no impact of metformin on colorectal cancer. In a cohort study conducted by Tsillidis KK et al in which 51,484 individuals were sub-grouped into metformin users and sulfonylurea users, and were followed up for 12 months. This study concluded that compared with the other group, initiators of metformin had a similar incidence of total cancer (HR 0.96; 95\% CI 0.89-1.04), colorectal (HR 0.92; 95\% CI 0.76-1.13), prostate (HR 1.02 ; 95\% CI 0.83-1.25), lung (HR 0.85; 95\% CI 0.681.07), postmenopausal breast cancer (HR 1.03; 95\% CI $0.82-1.31$ ) or any other cancer (Tsilidis et al., 2014). It is possible to argue that if the duration of follow up had been more than 12 months, the potential benefit of metformin might have been observed.

Lega et al showed that metformin was associated with a reduction in all-cause mortality (HR, 0.73; 95\% confidence intervals (CI), 0.64-0.83] and cancer-specific mortality (HR, 0.74; 95\% CI, 0.62-0.88)), and a significant reduction in mortality for colon cancer (four studies, HR, 0.65 ; 95\% CI, 0.56-0.76) but not for breast and prostate cancers (Lega et al., 2014).

In addition to the cohort studies described above, an experiment performed using cell culture, three cancer cell lines: HCT116, RKO and HT29 were grown in 10\% fetal bovine serum (FBS), treated with metformin (1 and $5 \mathrm{mM}$ ) with 5-amino-imidazol-4-carboxamide-1-b-4ribofuranoside (AICAR) $(5 \mathrm{mM})$ (AICAR is a AMPK activator) as a control. AICAR reduced cell viability, by $50-70 \%$ in the three cell lines, measured by MTT assay, whereas little decrease of cell viability was found in the three cell lines treated with metformin (Sui et al., 2014).
The cellular and molecular mechanisms for how metformin can suppress CRC

It is assumed that metformin interferes with cell growth and proliferation in many ways. It has anti-angiogenic and anti-proliferative effects (Zaafar et al., 2014). Metformin activates AMPK, which inhibits the mammalian target of rapamycin (mTOR) pathway. The mTOR pathway plays an important role in the cellular protein translational machinery and cell proliferation (Higurashi et al., 2012).

In addition, treatment with metformin selectively suppressed the tumor growth of HCT116 p53(-/-) xenografts. Following treatment with metformin, Buzzai et al. 2007, detected increased apoptosis in p53(-/-) tumor sections and an enhanced susceptibility of p53(-/-) cells to undergo apoptosis in vitro when subject to nutrient deprivation (Buzzai et al., 2007).

Metformin blocked the effect of the high-energy diet on tumour growth, reduced insulin levels, and attenuated the effect of diet on phosphorylation of AKT and expression of FASN. Furthermore, the administration of metformin led to the activation of AMPK, the inhibitory phosphorylation of acetyl-CoA carboxylase, the upregulation of BNIP3 and increased apoptosis as estimated by poly (ADP-ribose) polymerase (PARP) cleavage (SchoenAlgire et al., 2010) Figure 1.

\section{Conclusion}

In conclusion, the metabolic syndrome has been linked to an increased risk of colorectal cancer (CRC), and that treatment with metformin has a solid evidence supporting its beneficial role in improving the outcomes in CRC; through many mechanisms including anti-proliferative and anti-angiogenic effect. Still, many researches are to clarify the exact mechanisms of action of metformin on cancer cells, and the possible synergistic effect with other chemotherapeutic agents.

\section{References}

Aleksandrova K, Boeing H, Jenab M, et al (2011). Metabolic syndrome and risks of colon and rectal cancer: the European prospective investigation into cancer and nutrition study. Cancer Prev Res, 4, 1873-83.

Buzzai M, Jones R, Amaravadi R, et al (2007). Systemic treatment with the antidiabetic drug metformin selectively impairs p53-deficient tumor cell growth. Cancer Res, 67, 6745-52.

Cho Y, Ko B, Kim S, et al (2014). Does metformin affect the incidence of colonic polyps and adenomas in patients with type 2 diabetes mellitus? Intest Res, 12, 139-45.

Donohoe C, O'farrell N, Doyle S, Reynolds J (2014). The role of obesity in gastrointestinal cancer: evidence and opinion. Therap Adv Gastroenterol, 7, 38-50.

Giovannucci E, Pollak M, Platz E, et al (2000). A prospective study of plasma insulin-like growth factor-1 and binding protein-3 and risk of colorectal neoplasia in women. Cancer Epidemiol Biomarkers Prev, 9, 345-9.

Grimberg A \& Cohen P (2000). Role of insulin-like growth factors and their binding proteins in growth control and carcinogenesis. J Cell Physiol, 183, 1-9.

Handelsman Y, Leroith D, Bloomgarden Z, et al (2013). Diabetes 
and cancer--an AACE/ACE consensus statement. Endocr Pract, 19, 675-93.

Higurashi T, Takahashi H, Endo H, et al (2012). Metformin efficacy and safety for colorectal polyps: a double-blind randomized controlled trial. BMC Cancer, 12, 118.

Hillon P, Guiu B, Vincent J, Petit J (2010). Obesity, type 2 diabetes and risk of digestive cancer. Gastroenterol Clin Biol, 34, 529-33.

Hsu Y, Chiu H, Liou J, et al (2012). Glycated hemoglobin A1c is superior to fasting plasma glucose as an independent risk factor for colorectal neoplasia. Cancer Causes Control, 23, 321-8.

Huang HE, Yang YC, Wu JS, et al (2014). The relationship between different glycemic statuses and colon polyps in a Taiwanese population. J Gastroenterol, 49, 1145-51.

Inoue M, Tsugane S (2012). Insulin resistance and cancer: epidemiological evidence. Endocr Relat Cancer, 19, F1-8.

Jenkins P (2004). Acromegaly and cancer. Horm Res, 62, 108-15.

Kalaany N, Sabatini D (2009). Tumours with PI3K activation are resistant to dietary restriction. Nature, 458, 725-31.

Kanadiya M, Gohel T, Sanaka M, Thota P, Shubrook J (2013). Relationship between type-2 diabetes and use of metformin with risk of colorectal adenoma in an American population receiving colonoscopy. J Diabetes Complications, 27, 463-6.

Kaneko R, Nakazaki N, Tagawa T, et al (2014) A new index of abdominal obesity which effectively predicts risk of colon tumor development in female Japanese. Asian Pac J Cancer Prev, 15, 1005-10.

Khaw K, Wareham N, Bingham S, et al (2004). Preliminary communication: glycated hemoglobin, diabetes, and incident colorectal cancer in men and women: a prospective analysis from the European prospective investigation into cancer-Norfolk study. Cancer Epidemiol Biomarkers Prev, 13, 915-9.

Kim D (2012). [Obesity and gastrointestinal cancer-related factor]. Korean J Gastroenterol, 59, 8-15.

Larsson S, Wolk A (2007). Obesity and colon and rectal cancer risk: a meta-analysis of prospective studies. Am J Clin Nutr, 86, 556-65.

Lee D, Kim B, Lee J, et al (2012). The effect of metformin on responses to chemotherapy and survival in stage IV colorectal cancer with diabetes. Korean J Gastroenterol, 60, 355-61.

Lega I, Shah P, Margel D, et al (2014). The effect of metformin on mortality following cancer among patients with diabetes. Cancer Epidemiol Biomarkers Prev, 23, 1974-84.

Li W, Wang Q, Liu X, et al (2015). Combined use of vitamin D3 and metformin exhibits synergistic chemopreventive effects on colorectal neoplasia in rats and mice. Cancer Prev Res, 8, 139-48.

Mahmood K, Naeem M \& Rahimnajjad N (2013). Metformin: the hidden chronicles of a magic drug. Eur J Intern Med, 24, 20-6.

Mei Z, Zhang Z, Liu C, et al (2014). Survival benefits of metformin for colorectal cancer patients with diabetes: a systematic review and meta-analysis. PLoS One, 9, 91818.

Mulholland H, Murray L, Cardwell C \& Cantwell M (2009). Glycemic index, glycemic load, and risk of digestive tract neoplasms: a systematic review and meta-analysis. Am J Clin Nutr, 89, 568-76.

Na S \& Myung S (2012). [Obesity and colorectal cancer]. Korean J Gastroenterol, 59, 16-26.

Ogden C, Carroll M, Curtin L, et al (2006). Prevalence of overweight and obesity in the United States, 1999-2004. JAMA, 295, 1549-55.

Pais R, Silaghi H, Silaghi A, Rusu M \& Dumitrascu D (2009). Metabolic syndrome and risk of subsequent colorectal cancer. World J Gastroenterol, 15, 5141-8.

Rampal S, Yang MH, Sung J, et al (2014). Association between markers of glucose metabolism and risk of colorectal adenoma. Gastroenterol, 147, 78-87.

Renehan A, Roberts D, Dive C (2008). Obesity and cancer: pathophysiological and biological mechanisms. Arch Physiol Biochem, 114, 71-83.

Sanchez N, Stierman B, Saab S, et al (2012). Physical activity reduces risk for colon polyps in a multiethnic colorectal cancer screening population. BMC Res Notes, $\mathbf{5}, 312$.

Sandhu M, Dunger D, Giovannucci E (2002). Insulin, insulinlike growth factor-I (IGF-I), IGF binding proteins, their biologic interactions, and colorectal cancer. J Natl Cancer Inst, 94, 972-80.

Saydah SH, Platz EA, Rifai N, et al (2003). Association of markers of insulin and glucose control with subsequent colorectal cancer risk. Cancer Epidemiol Biomarkers Prev, 12, 412-8.

Schoenalgire C, Amrein L, Zakikhani M, Panasci L \& Pollak M (2010). Metformin blocks the stimulative effect of a high-energy diet on colon carcinoma growth in vivo and is associated with reduced expression of fatty acid synthase. Endocr Relat Cancer, 17, 351-60.

Spillane S, Bennett K, Sharp L, Barron T (2014). Metformin exposure and disseminated disease in patients with colorectal cancer. Cancer Epidemiol, 38, 79-84.

Sui X, Xu Y, Yang J, et al (2014). Use of metformin alone is not associated with survival outcomes of colorectal cancer cell but AMPK activator AICAR sensitizes anticancer effect of 5-fluorouracil through AMPK activation. PLoS One, 9, 97781.

Trevisan M, Liu J, Muti P, et al (2001). Markers of insulin resistance and colorectal cancer mortality. Cancer Epidemiol Biomarkers Prev, 10, 937-41.

Tsilidis K, Capothanassi D, Allen N, et al (2014). Metformin does not affect cancer risk: a cohort study in the U.K. Clinical Practice Research Datalink analyzed like an intention-totreat trial. Diabetes Care, 37, 2522-32.

Vu HT, Ufere N, Yan Y, et al (2014). Diabetes mellitus increases risk for colorectal adenomas in younger patients. World $J$ Gastroenterol, 20, 6946-52.

Wang X, Zhang Y (2014). Targeting mTOR network in colorectal cancer therapy. World J Gastroenterol, 20, 4178-88.

Warren R, Yuan H, Matli M, Ferrara N, Donner D (1996). Induction of vascular endothelial growth factor by insulinlike growth factor 1 in colorectal carcinoma. J Biol Chem, 271, 29483-8.

Williams A, Collard T, Perks C, et al (2000). Increased p53dependent apoptosis by the insulin-like growth factor binding protein IGFBP-3 in human colonic adenoma-derived cells. Cancer Res, 60, 22-7.

Yun K, Chang Y, Jung H, et al (2013). Impact of body mass index on the risk of colorectal adenoma in a metabolically healthy population. Cancer Res, 73, 4020-7.

Zaafar D, Zaitone S \& Moustafa Y (2014). Role of metformin in suppressing 1,2-dimethylhydrazine-induced colon cancer in diabetic and non-diabetic mice: effect on tumor angiogenesis and cell proliferation. PLoS One, 9, 100562.

Zaninotto P, Pierce M, Breeze E, De Oliveira C, Kumari M (2010). BMI and waist circumference as predictors of wellbeing in older adults: findings from the english longitudinal study of ageing. Obesity, 18, 1981-7.

Zhang P, Li H, Tan X, Chen L, Wang S (2013a). Association of metformin use with cancer incidence and mortality: a metaanalysis. Cancer Epidemiol, 37, 207-18.

Zhang Y, Guan M, Zheng Z, et al (2013b). Effects of metformin on CD133+ colorectal cancer cells in diabetic patients. PLoS 